\title{
Structure and stability of the $\operatorname{Si}(331)-(12 \times 1)$ surface reconstruction investigated with first-principles density functional theory
}

\author{
Corsin Battaglia* \\ Institut de Physique, Université de Neuchâtel, 2000 Neuchâtel, Switzerland \\ Giovanni Onida \\ Dipartimento di Fisica and European Theoretical Spectroscopy Facility (ETSF), Università di Milano, Via Celoria 16, \\ 20133 Milano, Italy \\ Katalin Gaál-Nagy \\ Dipartimento di Fisica and European Theoretical Spectroscopy Facility (ETSF), Università di Milano, Via Celoria 16, \\ 20133 Milano, Italy \\ Philipp Aebi \\ Institut de Physique, Université de Neuchâtel, 2000 Neuchâtel, Switzerland \\ (Received 4 February 2009; revised manuscript received 12 October 2009; published 3 December 2009)
}

\begin{abstract}
We recently proposed a structural model for the $\mathrm{Si}(331)-(12 \times 1)$ surface reconstruction containing silicon pentamers and adatoms as elementary structural building blocks. Using first-principles density functional theory we here investigate the stability of a variety of adatom configurations and determine the lowest-energy configuration. We also present a detailed comparison of the energetics between our model for $\mathrm{Si}(331)-(12$ $\times 1)$ and the adatom-tetramer-interstitial model for $\operatorname{Si}(110)-(16 \times 2)$, which shares the same structural building blocks.
\end{abstract}

DOI: $10.1103 /$ PhysRevB.80.214102

PACS number(s): 68.35.bg, 71.15.Mb

\section{INTRODUCTION}

High-index silicon surfaces are of interest not only from a fundamental point of view, but also as potential substrates for electronic device fabrication. The reconstructed $\mathrm{Si}(331)-(12$ $\times 1)$ surface is of particular importance since it is the only confirmed planar silicon surface with a stable reconstruction located between the (111) and (110) directions. Due to its large unit cell and its pronounced structural anisotropy it represents a promising template for the growth of lowdimensional heteroepitaxial nanostructures such as quantum dots and nanowires. ${ }^{1}$

Since its discovery more than 17 years ago ${ }^{2}$ several structural models containing dimers and adatoms as elementary structural building blocks have been proposed. ${ }^{3,4}$ We recently revealed the presence of an additional building block on the $\mathrm{Si}(331)-(12 \times 1)$ surface. ${ }^{5}$ Using scanning tunneling microscopy (STM) we were able to resolve for the first time rows of pentagon pairs running across the $\mathrm{Si}(331)$ surface. Very similar pentagons were observed earlier on the reconstructed $\mathrm{Si}(110)$ and $\mathrm{Ge}(110)$ surfaces. $^{6-8}$ Inspired by structural elements encountered on $\mathrm{Si}(113)$ and $\mathrm{Ge}(113)$ surfaces, ${ }^{9-11}$ An et $a l .{ }^{6}$ have proposed an adatom-tetramer-interstitial (ATI) model for the $\mathrm{Si}(110)-(16 \times 2)$ reconstruction containing silicon pentamers as building blocks which explain the pentagons observed in STM images. The stability of the ATI model has subsequently been tested theoretically by means of first-principles total energy calculations. ${ }^{12,13}$ Based on a detailed analysis of our experimental results and a comparison between the $\mathrm{Si}(110)$ and $\mathrm{Si}(331)$ surface, we proposed a structural model for the $\mathrm{Si}(331)-(12 \times 1)$ reconstruction containing silicon pentamers as essential building blocks. ${ }^{5}$
In order to account for the pentagons observed in our STM images, the model contains two pentamers per (12 $\times 1)$ unit cell, which saturate a certain number of energetically unfavorable dangling bonds of the bulk-truncated $\mathrm{Si}(331)$ surface. Some of the remaining dangling bonds are saturated by simple adatoms. The pentamer positions and bonding configurations were determined exclusively using experimental information and by comparing $\mathrm{Si}(331)$ to $\mathrm{Si}(110)$ as discussed in detail in Ref. 5. Although the available body of experimental results allows to narrow down the location of the adatoms, currently we do not have sufficient evidence to precisely determine the adatom positions on the surface solely based on information derived from experiment. In this paper we use first-principles total energy calculations to determine the precise adatom positions by finding the lowest-energy configuration among various alternative adatom configurations.

The paper is organized as follows: after briefly reviewing the methods used for the calculations, we focus on the bulktruncated $\mathrm{Si}(331)$ surface and compare its energetics to other silicon surfaces, which are known to be stabilized by surface reconstructions. In a second step pentamers are introduced and the consequences on the total energy are discussed. Then we consider a variety of candidate adatom configurations and determine the one with the lowest energy. At the end we discuss the impact of the remaining dangling bonds on the total energy.

\section{THEORY}

\section{A. Computational details}

Our results are based on first-principles calculations within density functional theory (DFT), ${ }^{16,17}$ using the local- 
density approximation (LDA) for the exchange-correlation functional. ${ }^{18,19}$ We adopt a plane-wave basis set and normconserving pseudopotentials, as implemented in the PWSCF code of the QUANTUM-ESPRESSO suite. ${ }^{20}$ Pseudopotentials for silicon and hydrogen are chosen in the von Barth style..$^{21,22}$

The $\mathrm{Si}(331)$ surface was simulated by means of an atomic slab and a repeated supercell, in order to recover a threedimensional periodicity. The slab thickness corresponds to 10 silicon double layers (DLs), and the periodic images are separated by $9.4 \AA$ of vacuum. Since each bulk DL contains 24 silicon atoms, our supercell counts 240 silicon atoms plus a number of silicon atoms at the top surface (varying from 0 to 16 depending on the specific reconstruction). The bottom surface of the slab is hydrogen passivated (with 36 hydrogen atoms).

Since we are interested in small energy differences, we adopt strict convergence criteria with respect to both the kinetic-energy cutoff and the sampling of the surface Brillouin zone. In particular, the inclusion of plane waves up to $544 \mathrm{eV}$ (40 Ry) and the use of a $x y$ shifted $3 \times 4 \times 1$ Monkhorst-Pack grid $^{23}$ for the $\mathbf{k}$ point sampling allowed us to ideally compute total energy differences with an accuracy of the order of $15 \mathrm{meV} / \mathrm{supercell}$. By taking into account the fact that our relaxed structures still show small residual forces (see below), we estimate our numerical accuracy on calculated surface energies to be better than $0.1 \mathrm{meV} / \AA^{2}$. All structures were optimized till the largest residual force on the mobile atoms was less than $0.026 \mathrm{eV} / \AA$ (0.001 Ry/ Bohr).

The hydrogen atom positions were determined in a first run by optimizing them on a bulk-terminated slab with fixed bulklike positions for silicon atoms, with top and bottom surfaces saturated by hydrogen. In all the following calculations we have taken hydrogen atomic positions at the bottom surface as fixed. In a second step, we inspected the decay of forces in the direction perpendicular to the surface, for a slab containing a nonrelaxed surface reconstruction. A nearly force-free bulk region is reached after the five topmost DLs. Thus, only the topmost five bulk silicon DL were allowed to relax together with the surface (reconstruction) atoms, where the inner DLs have been kept fixed. All results presented below are based on a slab relaxed in this way.

\section{B. Determination of the surface energy}

The surface energy $E_{\text {surf }}$ of a double-sided symmetric $(s)$ slab containing $N_{\mathrm{Si}}^{\mathrm{s}}$ silicon atoms can be written as ${ }^{14}$

$$
E_{\text {surf }}=\frac{1}{2}\left[E_{\text {tot }}^{\mathrm{Si}}\left(N_{\mathrm{Si}}^{\mathrm{s}}\right)-\mu_{\mathrm{Si}} N_{\mathrm{Si}}^{\mathrm{s}}\right],
$$

where $\mu_{\mathrm{Si}}$ is the chemical potential of silicon, being simply the bulk energy per atom at zero temperature. This value can be obtained from a simple bulk silicon calculation. $E_{\text {tot }}^{\mathrm{Si}}\left(N_{\mathrm{Si}}^{\mathrm{s}}\right)$ is the total energy of the slab. The factor $1 / 2$ comes from the fact that there are two identical surfaces. For a nonsymmetric slab, for which only the bottom surface is saturated by $\mathrm{H}$ atoms, we have

$$
E_{\text {surf }}+E_{\text {surf }}^{\mathrm{H}}=E_{\text {tot }}\left(N_{\mathrm{Si}}, N_{\mathrm{H}}\right)-\mu_{\mathrm{Si}} N_{\mathrm{Si}}-\mu_{\mathrm{H}} N_{\mathrm{H}} .
$$

Here, $E_{\text {surf }}^{\mathrm{H}}$ is the surface energy of the hydrogen saturated surface, $\mu_{\mathrm{H}}$ is the chemical potential for the hydrogen atoms, and $E_{\text {tot }}\left(N_{\mathrm{Si}}, N_{\mathrm{H}}\right)$ is the total energy of the slab whose bottom surface is saturated by $N_{\mathrm{H}}$ hydrogen atoms. Therefore, we have

$$
E_{\text {surf }}=E_{\text {tot }}\left(N_{\mathrm{Si}}, N_{\mathrm{H}}\right)-\mu_{\mathrm{Si}} N_{\mathrm{Si}}-\mu_{\mathrm{H}} N_{\mathrm{H}}-E_{\text {surf }}^{\mathrm{H}} .
$$

In order to avoid the determination of $\mu_{\mathrm{H}}$, we consider the following: the total energy of the slab can be written as

$$
E_{\text {tot }}\left(N_{\mathrm{Si}}, N_{\mathrm{H}}\right)=\frac{1}{2} E_{\text {tot }}^{\mathrm{H}}\left(\widetilde{N}_{\mathrm{Si}}^{\mathrm{s}}, 2 N_{\mathrm{H}}\right)+\frac{1}{2} E_{\mathrm{tot}}^{\mathrm{Si}}\left(N_{\mathrm{Si}}^{\mathrm{s}}\right),
$$

where $E_{\text {tot }}^{\mathrm{H}}$ is the total energy of a symmetric hydrogen saturated slab and $E_{\text {tot }}^{\mathrm{Si}}$ is the total energy of a symmetric slab containing the surface reconstruction. $N_{\mathrm{Si}}^{\mathrm{s}}$ and $\tilde{N}_{\mathrm{Si}}^{\mathrm{s}}$ differ only by the number of atoms used in the surface reconstruction. The value $E_{\text {tot }}^{\mathrm{H}}$ has been calculated in the preliminary run used for the determination of the hydrogen positions. The surface energy (per unit area) for the top surface carrying the surface reconstruction can then be determined using

$$
\gamma=E_{\text {surf }} / A=\left(E_{\mathrm{tot}}\left(N_{\mathrm{Si}}, N_{\mathrm{H}}\right)-\frac{1}{2} E_{\mathrm{tot}}^{\mathrm{H}}-N_{\mathrm{Si}}^{\mathrm{u}} \mu_{\mathrm{Si}}\right) / A,
$$

where $N_{\mathrm{Si}}^{\mathrm{u}}$ is the number of silicon atoms in the upper $(u)$ half slab and $A$ the surface area of the slab.

\section{RESULTS AND DISCUSSION}

\section{A. Bulk-truncated surface}

In order to define an energy reference for the following discussion we have determined the surface energy for the unrelaxed bulk-truncated $\mathrm{Si}(331)$ surface shown in Figs. 1(a) and 1(b). The value of $127.2 \mathrm{meV} / \AA^{2}$ may be compared in Table I with the values for other surface orientations.

The surface energy of a bulk-truncated surface can be estimated from the cohesive energy of silicon, which is $E_{c}$ $=4.6 \mathrm{eV}$ per atom. ${ }^{24}$ The cohesive energy of a solid is the energy per atom required to break the atoms of the solid into isolated atomic species. Since a bond is formed by two atoms and each silicon atom makes four bonds, the energy required to break a bond is half the cohesive energy, i.e., $E_{b}$ $=2.3 \mathrm{eV}$ per bond. ${ }^{25}$ The surface energy $\gamma$ can now be estimated by multiplying the bond energy $E_{b}$ by the density of broken bonds $n .{ }^{26}$ The latter is the number of dangling bonds $N$ per surface area $A, n=N / A$. Thus $\gamma=N E_{b} / 2 A=n E_{b} / 2$. The factor $1 / 2$ takes into account that two surfaces are created.

Note that the dangling bond density $n$ is not necessarily equal to the surface atom density (see values in parenthesis in Table I). This is due to the fact that a surface atom may either carry one or two dangling bonds. (111)-like surface atoms carry a single dangling bond, whereas (100)-like surface atoms carry two dangling bonds [see Figs. 2(a) and 3(a), respectively, in Ref. 27]. On the (113) surface, both types of surface atoms coexists, whereas on the (110) and the (331) surface only (111)-type surface atoms occur [see Fig. 5(a), 6(a), and 7(a), respectively, in Ref. 27]. 

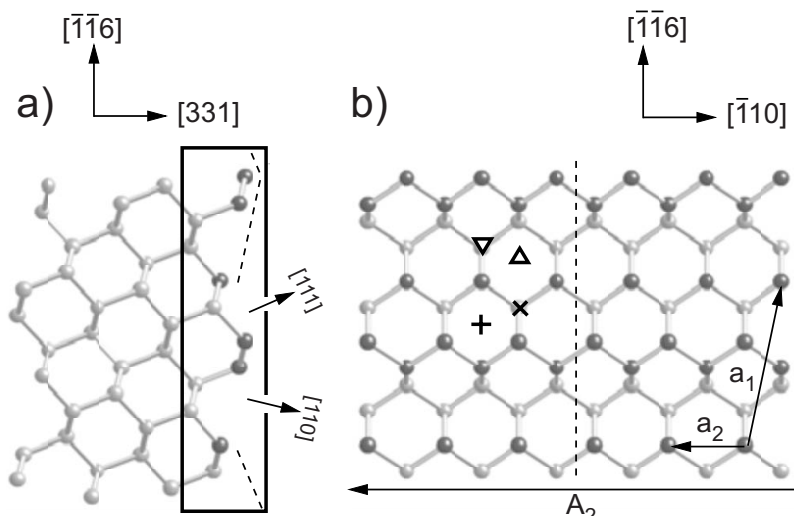

C)
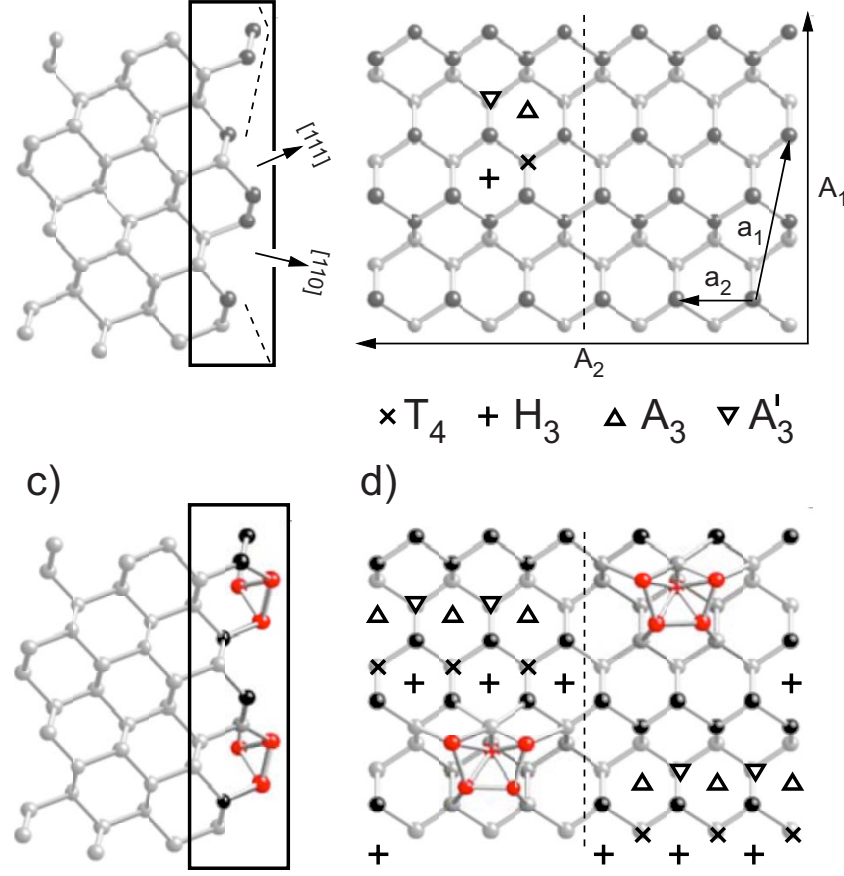

d)

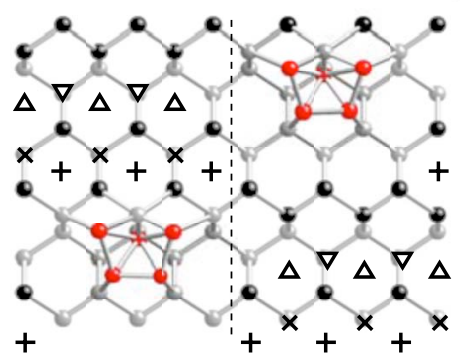

FIG. 1. (Color online) Side (a)/(c) and top view (b)/(d) of the unrelaxed bulk-truncated $\mathrm{Si}(331)-(12 \times 1)$ surface unit cell/ unrelaxed "pentamers only" model. The bulk directions are given. In (b)/(d) only the topmost atoms contained within the rectangle in (a)/(c) are shown. Subsurface atoms are shown in grey, undercoordinated surface atoms in black. Surface atoms forming the pentagon are called pentamer atoms (red in the online version). In (a) the $\mathrm{Si}(111)$ and $\mathrm{Si}(110)$ terraces composing the $\mathrm{Si}(331)$ surface are indicated. In $(b)$ the $(1 \times 1)$ and $(12 \times 1)$ surface unit cell vectors $\mathbf{a}_{1}$, $\mathbf{a}_{2}$ and $\mathbf{A}_{1}, \mathbf{A}_{2}$, respectively, are also drawn. The orientation of the glide plane is indicated by a dashed line. In (b) the four different types of adatom adsorption sites $T_{4}, H_{3}, A_{3}$, and $A_{3}^{\prime}$ are represented by markers. In (d) the $2 \times 12$ possible adatom positions after introduction of the two pentamers are shown.

In Table I we summarize estimated and calculated surface energies of unrelaxed bulk-truncated surfaces for a series of surface orientations. Although the simple estimate based on the cohesive energy fails to precisely reproduce the values determined with the help of first-principles methods, it correctly reproduces the energy ordering of the various surfaces. The estimated values tend to underestimate the surface energy for surfaces containing only (111)-type surface atoms, whereas they overestimate the surface energy for surfaces with (100)-type surface atoms. For $\mathrm{Si}(113)$, which contains both types of surface atoms, these two tendencies compensate resulting in the best agreement between estimated and calculated value.

From the theoretical point of view, the most stable surface is the one with the lowest surface energy. Consequently only structural models for the reconstructed surface with a surface energy lower than the bulk-truncated surface must be considered. Allowing the bulk-truncated surface to relax and determining the corresponding surface energy, we obtain an even more stringent condition on the surface energy for any valid candidate model. The relaxed structure of the bulk-truncated $\mathrm{Si}(331)$ surface is shown in Fig. 2(a). Note that although the relaxation was performed within the $(12 \times 1)$ supercell, it did not break the $(1 \times 1)$ periodicity within our numerical accuracy. The surface energy for the relaxed bulk-truncated surface is given in Table II along with the surface energies for other structures of the $\mathrm{Si}(331)$ and $\mathrm{Si}(110)$ surface discussed hereafter. Relaxation of the bulk-truncated $\mathrm{Si}(331)$ surface within the $(12 \times 1)$ unit cell results in a reduction of the surface energy of $16.3 \mathrm{meV} / \AA^{2}$, a value to be compared with the corresponding value of $21.3 \mathrm{meV} / \AA^{2}$ for $\mathrm{Si}(110)$ obtained by Stekolnikov et al. ${ }^{13}$ via relaxation of the bulktruncated $\mathrm{Si}(110)$ surface within the $(16 \times 2)$ cell.

\section{B. Pentamers}

The bulk-truncated $\mathrm{Si}(331)$ surface consists of alternating single and double rows of undercoordinated surface atoms running along the $[\overline{1} 10]$ direction [black atoms in Fig. 1(a) and $1(\mathrm{~b})]$. Each of the 36 (111)-type surface atoms per (12 $\times 1)$ unit cell carries one dangling bond, which renders the unreconstructed structure energetically unfavorable.

In our model, 10 out of the 36 dangling bonds are saturated by two silicon pentamers observed as pentagons in STM images (see inset in Fig. 3). Their integration onto the bulk-truncated $\mathrm{Si}(331)$ surface was discussed in detail in Ref.

TABLE I. Surface energies for unrelaxed bulk-truncated surfaces of various orientations. The numbers in parenthesis give the number of surface atoms and dangling bonds per $(1 \times 1)$ unit cell, respectively.

\begin{tabular}{ccccc}
\hline \hline $\begin{array}{c}\text { Surface } \\
\text { orientation }\end{array}$ & $\begin{array}{c}\text { Surface atom } \\
\text { density } \\
\left(\AA^{-2}\right)\end{array}$ & $\begin{array}{c}\text { Dangling bond } \\
\text { density } \\
\left(\AA^{-2}\right)\end{array}$ & $\begin{array}{c}\text { Estimated surface } \\
\text { energy } \\
\left(\mathrm{meV} / \AA^{2}\right)\end{array}$ & $\begin{array}{c}\text { Calculated surface } \\
\text { energy } \\
\left(\mathrm{meV}^{2} \AA^{2}\right)\end{array}$ \\
\hline$(111)$ & $0.078(1)$ & $0.078(1)$ & 90.1 & $113.6^{\mathrm{a}}$ \\
$(100)$ & $0.068(1)$ & $0.136(2)$ & 155.9 & $149.2^{\mathrm{a}}$ \\
$(113)$ & $0.082(2)$ & $0.123(3)$ & 141.0 & $137.9^{\mathrm{b}}$ \\
$(110)$ & $0.096(2)$ & $0.096(2)$ & 110.3 & $127.6^{\mathrm{a}}$ \\
$(331)$ & $0.093(3)$ & $0.093(3)$ & 107.3 & $127.2^{\mathrm{c}}$ \\
\hline \hline
\end{tabular}

\footnotetext{
Reference 14.

${ }^{\mathrm{b}}$ Reference 15 .

${ }^{\mathrm{c}}$ This work.
} 
a) bulk-truncated relaxed $\gamma=110.9$

b) pentamers only $\gamma=114.9$

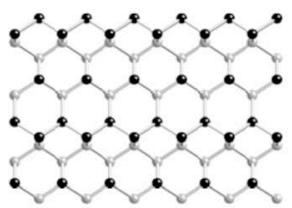

c) $\mathrm{T} 4 \gamma=111.8$
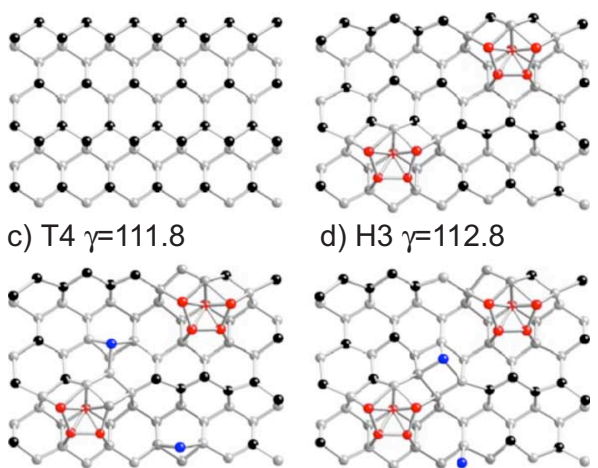

d) $\mathrm{H} 3 \gamma=112.8$

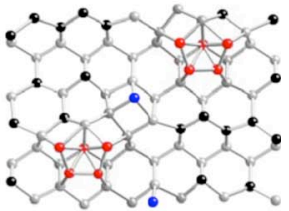

e) $A 3 \gamma=112.7$

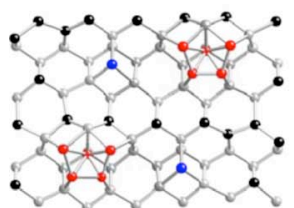

f) $\mathrm{T} 4 \mathrm{a} \gamma=111.2$

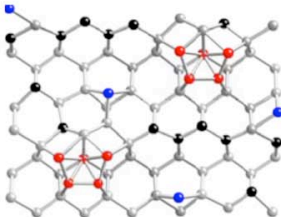

g) $T 4 b \gamma=110.0$

h) T4c $\gamma=110.2$

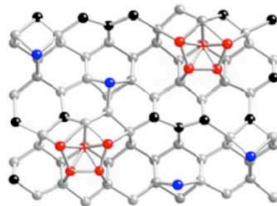

i) $\mathrm{T} 4 \mathrm{~d} \gamma=110.3$
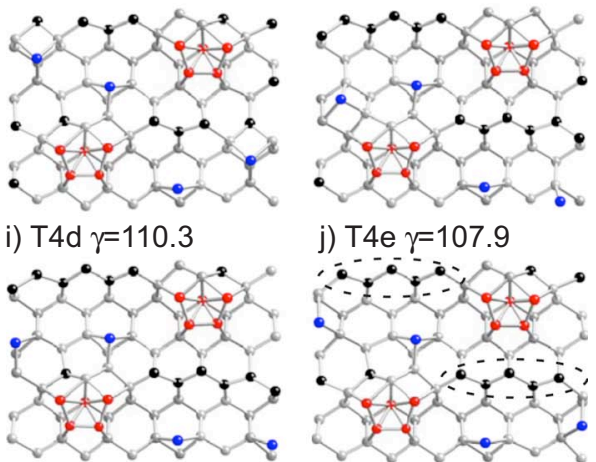

j) $\mathrm{T} 4 \mathrm{e} \gamma=107.9$

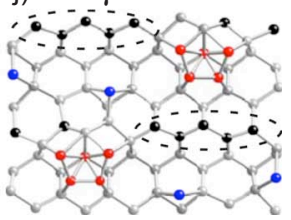

FIG. 2. (Color online) (a) Top view of the relaxed bulktruncated $\mathrm{Si}(331)-(12 \times 1)$ surface. (b) Top view of the relaxed "pentamers only" model. (c)-(j) Various candidate adatom configurations of the adatom-pentamer model. The surface energy $\gamma$ for each model is given in units of $\mathrm{meV} / \AA^{2}$.

5. It is important to note that the bulk-truncated $\mathrm{Si}(331)$ surface may be viewed as a highly stepped surface consisting of small alternating $\mathrm{Si}(111)$ and $\mathrm{Si}(110)$ terraces [see Fig. 1(a)]. This allows to anchor the pentamers on the Si(331) surface in exactly the same local binding configuration as on the $\mathrm{Si}(110)$ surface, where pentamers are also observed. Introducing two pentamers per unit cell as shown in Fig. 1(c) and 1(d) reduces the number of dangling bonds from 36 to 26 and lowers the surface energy [for the corresponding relaxed model shown in Fig. 2(b)] by $12.3 \mathrm{meV} / \AA^{2}$ with respect to the unrelaxed bulk-truncated surface (see Table II). This value is comparable with the reduction in $18.9 \mathrm{meV} / \AA^{2} \mathrm{ob}-$ tained when introducing four pentamers onto the $\mathrm{Si}(110)-(16 \times 2)$ surface [corresponding to the tetramerinterstitial (TI) model in Ref. 13, note that in the TI model the step is already included into the unit cell accounting for $1.0 \mathrm{meV} / \AA^{2}$ energy reduction]. However, the "pentamers only" models for both, $\mathrm{Si}(331)$ and $\mathrm{Si}(110)$, are not stable structures, since the relaxed bulk-truncated surface for both surfaces possesses a lower surface energy. This is not sur-

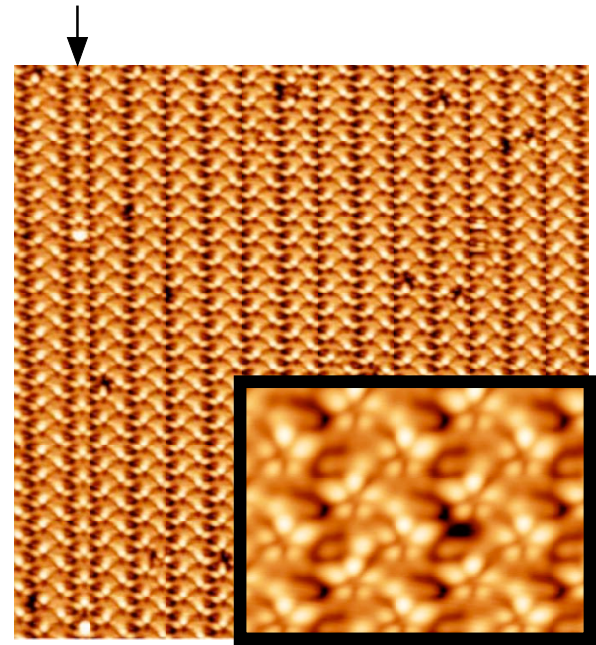

FIG. 3. (Color online) STM image of the Si(331)- $(12 \times 1)$ surface reconstruction with a single pentagon row marked by the arrow. Bias voltage $2.0 \mathrm{~V}$, set-point current $0.06 \mathrm{nA}$, temperature 77 $\mathrm{K}$, image size $35 \mathrm{~nm} \times 35 \mathrm{~nm}$, and inset image size $6 \mathrm{~nm}$ $\times 5 \mathrm{~nm}$.

prising, since the introduction of pentamers suggested by STM images, leaves a lot of dangling bonds unsaturated. So an additional building block is required to stabilize the structures containing pentamers.

\section{Adatoms}

In the ATI model for $\mathrm{Si}(110)-(16 \times 2)$, the pentamer structure is stabilized by adatoms. Among the various elementary structural building blocks observed in silicon surface reconstructions (see Ref. 27 for a review), adatoms impose themselves also as the best choice for $\mathrm{Si}(331)$ to saturate some of the remaining 26 dangling bonds for the following two reasons: firstly, as mentioned before, the bulk-truncated $\mathrm{Si}(331)$ surface may be viewed as being composed of small alternating (110) and (111) terraces. Since adatoms are observed on both the $\mathrm{Si}(110)$ and the $\mathrm{Si}(111)$ surface, the $\mathrm{Si}(331)$ consequently provides exactly the same local environment for ada-

TABLE II. Comparison between the surface energies of various surface structures of $\mathrm{Si}(331)$ and $\mathrm{Si}(110)$.

\begin{tabular}{|c|c|c|}
\hline \multirow[b]{2}{*}{ Structure } & \multicolumn{2}{|c|}{$\begin{array}{l}\text { Surface energy } \\
\left(\mathrm{meV} / \AA^{2}\right)\end{array}$} \\
\hline & $\operatorname{Si}(331)$ & $\operatorname{Si}(110)$ \\
\hline Bulk-truncated & $127.2^{\mathrm{a}}$ & $127.6^{\mathrm{d}}$ \\
\hline Bulk-truncated relaxed & $110.9^{\mathrm{a}}$ & $106.3^{\mathrm{c}, \mathrm{d}}$ \\
\hline Pentamers only & $114.9^{\mathrm{a}}$ & $108.7^{\mathrm{c}, \mathrm{d}}$ \\
\hline Adatoms and pentamers & $107.9^{\mathrm{a}}$ & $103.9^{\mathrm{c}, \mathrm{d}}$ \\
\hline Adatoms only & $104.6^{\mathrm{a}}$ & $101.4^{\mathrm{b}}$ \\
\hline
\end{tabular}

aThis work.

${ }^{\mathrm{b}}$ Reference 12.

${ }^{\mathrm{c}}$ Reference 13 .

${ }^{\mathrm{d}}$ Reference 14 . 
toms as on $\mathrm{Si}(110)$ and $\mathrm{Si}(111)$. Secondly adatoms allow to explain the additional protrusions seen in STM images, which can not be attributed to the pentamer atoms. ${ }^{5}$

An adatom requires three unsaturated surface atoms to be attached to. On the (111) terraces of the $\mathrm{Si}(331)$ surface, adatoms may occupy two possible sites indicated in Fig. 1(b). These geometries are distinguished as hollow $\left(H_{3}\right)$ and atop $\left(T_{4}\right)$ sites depending on whether the substrate atoms below the adatom are found in the fourth or second layer. In $H_{3}$ sites the adatom is threefold coordinated, in $T_{4}$ sites the adatom is approximately fourfold coordinated due to the substrate atom directly below in the second layer.

On the (110) terraces of the $\mathrm{Si}(331)$ surface, we can also distinguish between two different threefold coordinated adsorption sites depending on their orientation labeled $A_{3}$ and $A_{3}^{\prime}$ in Fig. 1(b). Note that on the bulk-truncated (110) surface these two sites become equivalent due to the mirror symmetry of the (110) surface along the [110] direction (not shown).

Careful inspection of Fig. 1(d) shows that after introducing two pentamers per unit cell there are exactly $2 \times 12$ possible positions for placing adatoms, the factor 2 is due to the glide plane symmetry along the $[\overline{1} \overline{1} 6]$ direction [dashed line in Fig. 1(d)]. Out of the $2 \times 12$ candidate adsorption sites, we identify $2 \times 3 \mathrm{~T}_{4}$ sites, $2 \times 4 \mathrm{H}_{3}$ sites, $2 \times 3 \mathrm{~A}_{3}$ sites and 2 $\times 2 A_{3}^{\prime}$ sites. This however does not imply that a maximum of 24 adatoms can be placed on the surface. The reason for this is as follows. After placing a first adatom on the surface, some surrounding candidate sites can not be occupied by an adatom anymore, since they shared the same dangling bond with the now occupied site.

A first pair of adatoms must be placed between two successive pentamers, in order to account for the clear protrusions seen in STM images. ${ }^{5}$ Due to the glide plane symmetry adatoms must always occur in pairs. There are only three candidate sites for this first adatom: a $T_{4}$ site [Fig. 2(c)], a $H_{3}$ site [Fig. 2(d)], and a $A_{3}$ site [Fig. 2(e)]. Using firstprinciples calculations we have tested these three possible adatom configurations and found that the $T_{4}$ site is the most stable of the three (surface energies for each model are also given in Fig. 2). This is not surprising, since $T_{4}$ sites are commonly favored over the $H_{3}$ sites on (111) surfaces such as in the $\mathrm{Si}(111)-(7 \times 7)$ reconstruction and the $\mathrm{Ge}(111)-c(2 \times 8)$ reconstruction (see Ref. 27 for a review of their structural models). In addition the $T_{4}$ adatom position reproduces best the protrusion observed by STM between two successive pentagons. ${ }^{5}$

Consequently, in order to reduce the large number of potential candidate adatom configurations to a computationally manageable number of models, we have restricted our further investigations to the subset of models containing the $T_{4}$ adatom pair. In a second step we have investigated all possible remaining positions for a second pair of adatoms. There are exactly five candidate configurations to be considered [shown in Fig. 2(f)-2(j)]. Using first-principles calculations we find that a $A_{3}$ adatom position (configuration T4e) gives the most stable configuration. The position of the $A_{3}$ adatom pair is also in agreement with light protrusions seen in STM images. ${ }^{5}$ After placing this $A_{3}$ adatom pair there is no more candidate site left on which one could place an additional adatom.

As discussed before, the introduction of two pentamers is not sufficient to obtain a lower surface energy than for the relaxed bulk-truncated surface. Inspection of Table II shows that adding adatoms to the pentamers allows to sufficiently lower the surface energy to render the reconstructed surface more stable than the relaxed bulk-truncated surface, for both the $\mathrm{Si}(331)$ and the $\mathrm{Si}(110)$ surfaces (reduction in surface energy by 7.0 and $4.8 \mathrm{meV} / \AA^{2}$, respectively, with respect to the "pentamers only" models).

\section{Rest atoms}

Introducing two $T_{4}$ and two $A_{3}$ adatoms per unit cell into our structural model for the $\mathrm{Si}(331)-(12 \times 1)$ reconstruction further reduces the number of dangling bonds from 26 to 14 [10 of the 36 dangling bonds are saturated by the pentamers, introducing 4 adatoms further eliminates $4 \times 3$ dangling bonds (here we do not count the dangling bond carried by each adatom)]. The remaining 14 surface atoms, which still carry a dangling bond, are so called rest atoms [black atoms in Fig. 2(j)].

Rest atoms are common structural building blocks accompanying adatoms. The adatom model for the Ge(111)-c(2 $\times 8)$ reconstruction is built from four adatoms and four rest atoms. For this case, we have an ideal one-to-one correspondence between adatoms and rest atoms. Each adatom delivers one electron from its dangling bond to fill the dangling bond of the rest atom resulting in a further decrease in the surface energy. In contrast, in the famous dimer-adatomstacking fault (DAS) model of the $\mathrm{Si}(111)-(7 \times 7)$ reconstruction, there are 12 adatoms, but only six rest atoms plus the corner hole atom, which behaves similarly to a rest atom. In this case, using a simple ionic picture, we have seven electrons which are transferred from the adatoms into the rest atom plus corner hole atom states, but five electrons remain in the adatoms bands. For the ATI model of the $\mathrm{Si}(110)-(16 \times 2)$ reconstruction, there are eight adatoms and 12 rest atoms. So even when transferring the eight electrons from the adatoms to the rest atoms, only a fraction of the rest atoms may saturate their dangling bonds. Similarly in our model, with four adatoms and 14 rest atoms, we expect some remaining unsaturated rest atoms.

The unequal adatom:rest atom ratio between the ATI model $(8: 12=0.67)$ of $\mathrm{Si}(110)$ and our model $(4: 14=0.29)$ of $\mathrm{Si}(331)$ might on the first view explain why the ATI model exhibits a slightly lower surface energy than our model (energy difference $4.0 \mathrm{meV} / \AA^{2}$, see Table II). Reinspection of Table II however reveals that this energy difference between $\mathrm{Si}(331)$ and $\mathrm{Si}(110)$, whose corresponding unrelaxed bulktruncated surfaces have almost the same energy (energy difference only $0.4 \mathrm{meV} / \AA^{2}$ ), is already present for the respective "pentamers only" models (energy difference between "pentamers only" models $6.2 \mathrm{meV} / \AA^{2}$ ). It is also interesting to note that the introduction of the adatoms leads to a larger reduction of the surface energy for $\mathrm{Si}(331)\left(7.0 \mathrm{meV} / \AA^{2}\right)$ than for $\mathrm{Si}(110)\left(4.8 \mathrm{meV} / \AA^{2}\right)$ with respect to the "pentamers only" models. 
TABLE III. Surface energies for silicon surface reconstructions.

\begin{tabular}{lc}
\hline \hline Reconstruction & $\begin{array}{c}\text { Surface energy } \\
\left(\mathrm{meV} / \AA^{2}\right)\end{array}$ \\
\hline $\mathrm{Si}(111)-(7 \times 7)$ & $84.9^{\mathrm{a}}$ \\
$\mathrm{Si}(100)-c(4 \times 2)$ & $88.0^{\mathrm{a}}$ \\
$\mathrm{Si}(113)-(3 \times 2)$ & $87.9^{\mathrm{b}} / 87.4^{\mathrm{a}}$ \\
$\mathrm{Si}(110)-(16 \times 2)$ & $103.9^{\mathrm{a}, \mathrm{c}}$ \\
$\mathrm{Si}(331)-(12 \times 1)$ & $107.9^{\mathrm{d}}$ \\
\hline
\end{tabular}

${ }^{\text {aReference } 14}$

${ }^{\mathrm{b}}$ Reference 28 .

${ }^{\mathrm{c}}$ Reference 13.

${ }^{\mathrm{d}}$ This work.

Introducing hypothetically an additional adatom pair into our model for $\mathrm{Si}(331)$ would improve its adatom:rest atom ratio from $4: 14=0.29$ to $6: 8=0.75$. The introduction of the first pair of adatoms $\left(T_{4}\right)$ accounts for a reduction of $3.1 \mathrm{meV} / \AA^{2}$ of the surface energy. Adding a second pair of adatoms $\left(A_{3}\right)$ to the first pair allowed to reduce the surface energy by $3.9 \mathrm{meV} / \AA^{2}$. Following this tendency, by assuming an energy gain of $3-4 \mathrm{meV} / \AA^{2}$ for the introduction of a hypothetical third pair of adatoms into our model, suggests that the surface energy of $\mathrm{Si}(331)$ comes to lie close to the value determined for $\mathrm{Si}(110)$ by Stekolnikov et al. ${ }^{13}$ However, note that the rest atom geometry of T4e does not allow the introduction of an additional adatom pair. When considering the subset of T4x models (with $x=\mathrm{a}-\mathrm{e}$ ), only configurations $\mathrm{T} 4 \mathrm{a}$ and $\mathrm{T} 4 \mathrm{~b}$ admit a pair of free adatom sites, resulting in configuration $\mathrm{T} 4 \mathrm{a}+\mathrm{b}$, with a pair of $\mathrm{H}_{3}$ adatoms (as in T4a) and a nearby pair of $A_{3}^{\prime}$ adatoms (as in T4b) between the double pentagon rows, besides the initial $T_{4}$ adatom pair. This configuration was discarded because only one adatom pair is observed between the double pentagon rows in our high-resolution STM images. ${ }^{5}$ In addition the calculated surface energy of $111.4 \mathrm{meV} / \AA^{2}$ for the relaxed $\mathrm{T} 4 \mathrm{a}+\mathrm{b}$ configuration is significantly larger than the surface energy for the $\mathrm{T} 4 \mathrm{e}$ configuration $\left(107.9 \mathrm{meV} / \AA^{2}\right)$. This excludes the $\mathrm{T} 4 \mathrm{a}+\mathrm{b}$ configuration from the list of possible candidates.

Several more three adatom pair configurations are possible when studying $\mathrm{H} 3 \mathrm{x}$ (8/7 possible two/three adatom pair configurations) or $\mathrm{A} 3 \mathrm{x}$ configurations (5/1 possible two/three adatom pair configurations). Even more possibilities may exist when allowing the placement of the first adatom pair on a different site. Here we focused on the family of T4x models, whose selection was motivated and justified in section $\mathrm{C}$. However, before testing other potential configurations, further experimental hints on the position of the adatoms from complementary experimental techniques are necessary to assist in the search for a possible lower-energy configuration.

\section{E. Adatoms only configurations}

When compared to the surface energies of reconstructions on surfaces with other orientations listed in Table III, the models for the $\mathrm{Si}(110)-(16 \times 2)$ and the $\mathrm{Si}(331)-(12 \times 1)$ reconstruction have surface energies which are of the order of
$20 \mathrm{meV} / \AA^{2}$ higher than the surface energies for $\mathrm{Si}(111)-(7$ $\times 7), \operatorname{Si}(100)-c(4 \times 2)$, and $\operatorname{Si}(113)-(3 \times 2)$. As it was already mentioned by Stekolnikov et al. ${ }^{13}$ in the context of the ATI model for $\mathrm{Si}(110)-(16 \times 2)$, a more complex reconstruction model cannot be excluded.

Until now we have limited our search for possible candidate structures to configurations containing two pentamers as elementary structural building blocks, since these pentamers are able to explain the pentagons experimentally observed in STM images. In order to sample configurations without pentagons, we performed calculations for various "adatoms only" configurations, i.e., configurations without pentamers containing only adatoms. Among the tested configuration, we find two "adatoms only" configurations, equivalent to T4 (107.6 meV/ $\left.\AA^{2}\right)$ and T4e $\left(104.6 \mathrm{meV} / \AA^{2}\right)$, but without pentamers, which exhibit surface energies lower than the $\mathrm{T} 4 \mathrm{e}$ configuration containing the two experimentally observed pentamers $\left(107.9 \mathrm{meV} / \AA^{2}\right)$. A similar situation is actually also observed for the $\operatorname{Si}(110)$ surface. There a $(3 \times 1)$ "adatoms only" configuration (101.4 $\left.\mathrm{meV}^{\circ} \AA^{2}\right)$ (Ref. 12) is found to have a surface energy lower than the full Si(110)-(16×2) ATI model $\left(103.9 \mathrm{meV} / \AA^{2}\right)$. All these "adatoms only" configurations are not able to explain the pentagons observed in STM images (see Fig. 3) and are therefore unphysical.

Several explanations are possible. Possibly the $\mathrm{Si}(331)-(12 \times 1)$ and $\mathrm{Si}(110)-(16 \times 2)$ reconstructions do not represent equilibrium surface phases. Stekolnikov et al. ${ }^{13}$ argue that kinetic barriers may play a role. A kinetic barrier is known to exist between $\operatorname{Si}(111)-(2 \times 1)$, obtained after cleaving, which converts into $\mathrm{Si}(111)-(7 \times 7)$ upon annealing. ${ }^{29}$ However, the thermal preparation cycle employed to obtain the $\operatorname{Si}(331)-(12 \times 1)$ surface (see Fig. 2 in Ref. 1) does not support an explanation based on kinetic barriers.

Furthermore surface doping could influence the relative stability between the various structural configurations. Experimental studies so far have been carried out on $p$-type $\mathrm{Si}(331)$ surfaces (mostly boron-doped). On the $\mathrm{Si}(111)$ surface, boron atoms possess a tendency to segregate and accumulate at the surface during high-temperature annealing. ${ }^{30}$ For prolonged annealing, boron atoms are even capable of inducing a phase transition between the $\mathrm{Si}(111)-(7 \times 7)$ and a boron induced $\mathrm{Si}(111)-(\sqrt{3} \times \sqrt{3})$ reconstruction. In this structure the boron atoms sit directly below $\mathrm{T} 4$ silicon adatoms. $^{31}$ The stability of this configuration is due to the relief of subsurface strain by the short boron-silicon bonds and the passivation of the surface obtained through charge transfer from the silicon adatom to the substitutional boron atom. Substitutional subsurface boron atoms could therefore also be considered for passivation of at least some of the 14 rest atoms or the adatoms of the T4e model. However, experiments on $\mathrm{Si}(111)$ samples with a similar doping level as for the $\mathrm{Si}(331)$ samples used in our previous study, ${ }^{1,5}$ do indicate that the effect of boron atoms at the surface is not of relevance for the stability of the $\mathrm{Si}(331)-(12 \times 1)$ reconstruction. In addition, we also observed alternating facets of $\operatorname{Si}(331)-(12 \times 1)$ and $\operatorname{Si}(111)-(7 \times 7)$ on well-annealed $n$-type phosphor-doped $\mathrm{Si}(553)$ samples, so that we can rule out boron atoms for explaining the stability of the $\operatorname{Si}(331)-(12 \times 1)$ surface. 
Lateral compressive strain has been shown to be able to convert the $\mathrm{Ge}(111)-(2 \times 8)$ "adatoms only" reconstruction into a $\mathrm{Ge}(111)-(7 \times 7)$ reconstruction with the same structure as the $\mathrm{Si}(111)-(7 \times 7)$ surface. ${ }^{32}$ Bechstedt et al. ${ }^{29}$ demonstrated within DFT and the LDA that these two structures are energetically degenerate. A possible indication that lateral strain is present in the $\mathrm{Si}(331)-(12 \times 1)$ reconstruction is the occurrence of single rows of pentagons as shown by the arrow in the STM image in Fig. 3. This topological defect possibly allows relaxation of the accumulated strain. We have therefore performed a calculation in which not only the atoms were allowed to relax but also the lateral lattice constants of the slab. For T4e with and without pentagons we obtain surface energies of 106.7 and $103.4 \mathrm{meV} / \AA^{2}$ respectively. These values must be compared to the previous values of 107.9 and $104.6 \mathrm{meV} / \AA^{2}$ obtained when only the atoms are allowed to relax. For both configurations the energy reduction upon relaxation of the lateral lattice constants is $1.2 \mathrm{meV} / \AA^{2}$ and thus the relative stability of these two structures is not modified.

Possibly model T4e we proposed for the $\mathrm{Si}(331)-(12$ $\times 1)$ reconstruction is still missing an important ingredient. However, a possible yet unknown elementary structural building block hidden between the pentamers has still not been revealed by any experiment. Without further experimental input, we can only speculate about its nature. As a starting point for further improvement of our model, one may reconsider the adatoms and rest atoms of model $\mathrm{T} 4 \mathrm{e}$. The four adatoms of model T4e provide four electrons which may be used to passivate four rest atoms. 10 unsaturated rest atoms remain. Inspection of Fig. 2(j) reveals that model T4e contains two rest atom chains with five rest atoms connected to each other by one bond (within the dotted ellipses). These chain segments have a certain resemblance with the Seiwatz chains, an elementary structural building block for atomic chains on the $\mathrm{Si}(111)$ surface. On $\mathrm{Si}(111)$, Seiwatz chains are known to be stabilized by divalent adsorbates (see Ref. 33 for a review). However, based on the current experimental data it is not clear if and how this building block could be incorporated into our model.

Nevertheless, the advantage of our method for developing a structural model for $\mathrm{Si}(331)-(12 \times 1)$ consists here in integrating elementary structural building blocks such as pentamers and adatoms identified on the (110) and (111) silicon surfaces $^{27}$ onto the bulk-truncated $\mathrm{Si}(331)$ surface. The con- sistency of the pentamer building block with all currently available experimental data on $\mathrm{Si}(331)-(12 \times 1)$, but also on $\mathrm{Si}(110)-(16 \times 2)$, represents further indirect evidence in favor of the two structural models. It should be noted that our model for $\mathrm{Si}(331)$ is far from a simple extension of the $\mathrm{Si}(110)$ surface to its vicinals. The pentagons on $\mathrm{Si}(110)$ (see Fig. 6 in Ref. 27) are arranged in pairs pointing in opposite directions, whereas on $\mathrm{Si}(331)$ pairs point in the same direction. Also the direction of the double pentagon rows appears to be completely unrelated between the two surfaces. Using pentamers and adatoms we succeeded in combining these seemingly conflicting experimental observations in a coherent picture. Since we also found an energetically more favorable "adatoms only" configuration which is in disagreement with experimental STM images, further studies are necessary.

\section{SUMMARY AND CONCLUSIONS}

Using first-principles density functional theory we studied the stability of the structural model for the $\mathrm{Si}(331)-(12 \times 1)$ surface reconstruction, which we recently proposed. It contains two silicon pentamers and a number of silicon adatoms. We determined the energetically most favorable adatom configuration among a variety of candidate structures. We find that a configuration with a pair of $T_{4}$ adatoms and a second pair of $A_{3}$ adatoms results in the lowest energy. We discussed the implications of the rest atoms on the energy and compared our model to other silicon surface reconstructions. However, we also find that an "adatoms only" configuration without pentamers but only a pair of $T_{4}$ and $A_{3}$ adatoms results in a lower energy. Additional experimental input is required to resolve this contradiction.

\section{ACKNOWLEDGMENTS}

Stimulating discussions with Andrey Stekolnikov, Claude Monney, Clément Didiot, Eike Schwier, and Michael Gunnar Garnier are gratefully acknowledged. Skillful technical assistance was provided by our workshop and electric engineering team. This work was supported by the Fonds National Suisse pour la Recherche Scientifique through Div. II and the Swiss National Center of Competence in Research MaNEP. We acknowledge support by the European Community through the "Nanoquanta NoE" and "ETSF-I3" projects (Grant No. NMP4-CT-2004-500198 and Grant Agreement No. 211956) for the theoretical results, which are an outcome of the ETSF User Project No. 94.

\footnotetext{
*Present address: Institute of Microengineering (IMT), Photovoltaics and Thin Film Electronics Laboratory, Ecole Polytechnique Fédérale de Lausanne (EPFL), 2000 Neuchâtel, Switzerland. corsin.battaglia@epfl.ch

${ }^{\dagger}$ Present address: Dipartimento di Fisica and European Theoretical Spectroscopy Facility (ETSF), Università di Milano, Via Monte Velino 17, 20137 Milano, Italy.

*Present address: Department of Physics, Université de Fribourg, 1700 Fribourg, Switzerland. http://physics.unifr.ch/en/page/87/

${ }^{1}$ C. Battaglia, C. Monney, C. Didiot, E. F. Schwier, M. Mariotti,
}

M. G. Garnier, and P. Aebi, arXiv:0809.4967, AIP Conf. Proc. (to be published).

${ }^{2}$ J. Wei, E. D. Williams, and R. L. Park, Surf. Sci. Lett. 250, L368 (1991).

${ }^{3}$ B. Z. Olshanetsky, S. A. Teys, and I. G. Kozhemyako, Phys. Low-Dimens. Struct. 11-12, 85 (1998).

${ }^{4}$ Z. Gai, R. G. Zhao, T. Sakurai, and W. S. Yang, Phys. Rev. B 63, 085301 (2001).

${ }^{5}$ C. Battaglia, K. Gaál-Nagy, C. Monney, C. Didiot, E. F. Schwier, M. G. Garnier, G. Onida, and P. Aebi, Phys. Rev. Lett. 
102, 066102 (2009).

${ }^{6}$ T. An, M. Yoshimura, I. Ono, and K. Ueda, Phys. Rev. B 61, 3006 (2000).

${ }^{7}$ T. Ichikawa, T. Sueyosi, T. Saot, M. Iwatsuki, F. Udagwa, and I. Sumita, Solid State Commun. 93, 541 (1995).

${ }^{8}$ Z. Gai, R. G. Zhao, and W. S. Yang, Phys. Rev. B 57, R6795 (1998).

${ }^{9}$ J. Dabrowski, H.-J. Müssig, and G. Wolff, Phys. Rev. Lett. 73, 1660 (1994).

${ }^{10}$ A. Laracuente, S. C. Erwin, and L. J. Whitman, Phys. Rev. Lett. 81, 5177 (1998).

${ }^{11}$ A. A. Stekolnikov, J. Furthmüller, and F. Bechstedt, Phys. Rev. B 67, 195332 (2003).

${ }^{12}$ A. A. Stekolnikov, J. Furthmüller, and F. Bechstedt, Phys. Rev. B 70, 045305 (2004).

${ }^{13}$ A. A. Stekolnikov, J. Furthmüller, and F. Bechstedt, Phys. Rev. Lett. 93, 136104 (2004).

${ }^{14}$ A. A. Stekolnikov, J. Furthmüller, and F. Bechstedt, Phys. Rev. B 65, 115318 (2002).

${ }^{15}$ A. A. Stekolnikov, J. Furthmüller, and F. Bechstedt, Phys. Rev. B 68, 205306 (2003).

${ }^{16}$ P. Hohenberg and W. Kohn, Phys. Rev. 136, B864 (1964).

${ }^{17}$ W. Kohn and L. J. Sham, Phys. Rev. 140, A1133 (1965).

${ }^{18}$ J. P. Perdew and A. Zunger, Phys. Rev. B 23, 5048 (1981).
${ }^{19}$ D. M. Ceperley and B. J. Alder, Phys. Rev. Lett. 45, 566 (1980).

${ }^{20} \mathrm{http}$ ://www.pwscf.org.

${ }^{21}$ U. von Barth and R. Car (unpublished).

${ }^{22}$ A. Dal Corso, S. Baroni, R. Resta, and S. de Gironcoli, Phys. Rev. B 47, 3588 (1993).

${ }^{23}$ H. J. Monkhorst and J. D. Pack, Phys. Rev. B 13, 5188 (1976).

${ }^{24}$ B. Farid and R. W. Godby, Phys. Rev. B 43, 14248 (1991).

${ }^{25}$ W. A. Harrison, Electronic Structure and Properties of Solids (Freeman, San Francisco, 1980).

${ }^{26}$ J. Dabrowski and H. J. Müssig, Silicon Surfaces and Formation of Interfaces (World Scientific, Singapore, 2000).

${ }^{27}$ C. Battaglia, K. Gaál-Nagy, C. Monney, C. Didiot, E. F. Schwier, M. G. Garnier, G. Onida, and P. Aebi, J. Phys.: Condens. Matter 21, 013001 (2009).

${ }^{28}$ K. Gaál-Nagy and G. Onida, Phys. Rev. B 75, 155331 (2007).

${ }^{29}$ F. Bechstedt, A. A. Stekolnikov, J. Furthmüller, and P. Käckell, Phys. Rev. Lett. 87, 016103 (2001).

${ }^{30}$ M. Berthe et al., Phys. Rev. Lett. 97, 206801 (2006).

${ }^{31}$ I.-W. Lyo, E. Kaxiras, and P. Avouris, Phys. Rev. Lett. 63, 1261 (1989).

${ }^{32}$ H.-J. Gossmann, J. C. Bean, L. C. Feldman, E. G. McRae, and I. K. Robinson, Phys. Rev. Lett. 55, 1106 (1985).

${ }^{33}$ C. Battaglia, H. Cercellier, C. Monney, L. Despont, M. G. Garnier, and P. Aebi, J. Phys.: Conf. Ser. 100, 052078 (2008). 